\title{
Soil chemical properties, 'Guanximiyou' pummelo leaf mineral nutrient status and fruit quality in the southern region of Fujian province, China
}

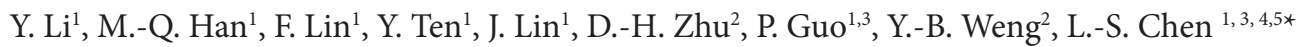

${ }^{1}$ College of Resource and Environmental Science, Fujian Agriculture and Forestry University, Fuzhou 350002, PR China. 'Agricultural Bureau of Pinghe County, Zhangzhou 363700, China. ${ }^{3}$ Institute of Horticultural Plant Physiology, Biochemistry and Molecular Biology, Fujian Agriculture and Forestry University, Fuzhou 350002, China. ${ }^{4}$ Fujian Key Laboratory for Plant Molecular and Cell Biology, Fujian Agriculture and Forestry University, Fuzhou 350002, China. ${ }^{5}$ The Higher Educational Key Laboratory of Fujian Province for Soil Ecosystem Health and Regulation, Fujian Agriculture and Forestry University, Fuzhou 350002, China *Corresponding author: lisongchen2002@hotmail.com

\begin{abstract}
Three hundred and nineteen 'Guanximiyou' pummelo (Citrus grandis) orchards from Pinghe county, the southern region of Fujian province, China, were selected for this study. The objectives were to determine (i) the soil and leaf nutrient status, (ii) the relationships between leaf mineral elements and the corresponding soil elements, and (iii) the relationships between fruit quality and mineral nutrients. The results showed that soil acidification was a major problem in these orchards, with an average $\mathrm{pH}$ of 4.34 . Soil acidification affected the availability of soil N, P, Ca, Mg, S, B, Cu and $\mathrm{Zn}$ and the levels of organic matter (OM) and cation exchange capacity (CEC), thus inducing soil and leaf nutrient imbalance. Indeed, severe nutrient imbalance existed in these orchard soils. $77.4 \%$ and $65.8 \%$ of soils were sub-optimum in exchangeable $\mathrm{Mg}$ and $\mathrm{Ca}$, while $96.6 \%$ and $82.1 \%$ of soils were super-optimum in available $\mathrm{S}$ and $\mathrm{P}$, respectively. Besides, severe nutrient deficiencies and excesses co-existed in leaves. $46.8 \%$ and $35.6 \%$ of leaves were deficient in $\mathrm{N}$ and $\mathrm{Mg}$, while $74.8 \%$ and $70.4 \%$ of leaves were excess in $\mathrm{B}$ and $\mathrm{Cu}$, respectively. Regressive analysis showed that leaf content of mineral elements was poorly related with the available content of the corresponding soil elements, respectively. In some orchards, severe juice sac granulation, an important factor affecting fruit quality, was observed. Regressive analysis indicated that $\mathrm{Mg}, \mathrm{S}$, $\mathrm{Cu}$ and $\mathrm{Mn}$ played a role in juice sac granulation of fruits. In conclusion, soil acidification might lead to severe soil nutrient imbalance, thus inducing leaf nutrient imbalance, eventually impairing fruit quality parameters such sac granulation.
\end{abstract}

Keywords: Citrus grandis, granulation, nutrient imbalance, soil acidification 


\section{Introduction}

Citrus is the leading fruit crop in the world, citrus production and consumption have grown strongly since the mid-1980s (FAOSTAT data). In 2013, over 140 countries produced citrus fruits. Main producing countries are China, Brazil and the United States (FAOSTAT data). In 2011, there are approximately 2.29 million ha of citrus planted in 19 provinces of China and a yield of $2944 \times 10^{4} \mathrm{t}$ (Ministry of Agriculture P.R.C, 2012). Pummelo (Citrus grandis) is the third major types of citrus after Citrus reticulata (i.e., tangerines, mandarins, clementines and satsumas) and oranges (Citrus sinensis) with a yield of $320 \times 10^{4} \mathrm{t}$ (Ministry of Agriculture P.R.C, 2012). In China, the most famous area for pommelo production is Pinghe county, Fujian province. In Pinghe, 'Guanximiyou' pummelo has been planted for more than 500 years and was used to be a tribute for royal. By 2013, total production of 'Guanximiyou' pummelo reached over $4 \times 10^{4}$ ha with an annual production of over $120 \times 10^{4} \mathrm{t}(\mathrm{Lu}, 2013)$. Recently, 'Guanximiyou' pummelo fruit quality displayed a down trend. Both maximum fruit quality and yield will occur only in the presence of optimum nutrient balance and intensity. Low fruit quality is often associated with poor soil fertility and poor nutrient management (Zhuang, 1994). Adequate nutrient management would never have been possible without the knowledge of soil fertility and plant nutrition status.

Soil testing, which is crucial for evaluating soil fertility, is believed to be a necessary complement to leaf analysis for citrus fertilizer recommendations (Du Plessis et al., 1977). Citrus growers should maintain soil fertility, thus preventing nutrient deficiencies and excesses, since both affect the yield and quality of citrus fruits. Soil fertility is mainly determined by various chemical properties such as soil $\mathrm{pH}$, cation exchange capacity (CEC), content of organic matter $(\mathrm{OM})$ and mineral nutrients.
Citrus stores significant amounts of mineral nutrients in tree biomass, part of which can be redistributed mainly to developing organs such as fruits and young leaves. Evidence shows that leaf content of some mineral elements does not correlate with the available content of the corresponding soil elements, respectively (Huang et al., 2001; Tang et al., 2013). Better reliability of leaf analysis over soil analysis has diverted towards finding the cause and effect relationship on fruit quality of pummelo.

To date, the premier fruit quality parameters of pummelo which guide the marketability, through global research data on pummelo is meager. During 19981999, Huang et al. (2001) investigated mineral element content in orchard soils and leaves of 'Guanximiyou' pummelo, and soil $\mathrm{pH}$ and OM. Unfortunately, fruit quality parameters were not determined in this study. In addition, soil acidification can occur in the past decade due to acid deposition and some farming practices such as improper fertilization, intensive agriculture and monoculture (Guo et al., 2010), thus affecting nutrient balance and intensity.

In this study, we measured soil $\mathrm{pH}, \mathrm{CEC}, \mathrm{OM}$, content of mineral element in soils and leaves, and fruit quality parameters of 'Guanximiyou' pummelo grown in the southern region of Fujian province, China. The objectives of this study were to understand (i) the soil and leaf nutrient status, (ii) the relationships between leaf mineral elements and the corresponding soil elements, and (iii) the relationships between fruit quality and mineral nutrients.

\section{Materials and Methods}

Three hundred and nineteen 'Guanximiyou' pummelo orchards from ten townships (Jiufeng, Qiling, Xiazhai, Wenfeng, Guoqiang, Xiaoxi, Shange, Nansheng, Banzi 
and Luxi) were selected for this study. The orchards were located in Pinghe county $\left(24^{\circ} 02^{\prime}-24^{\circ} 35^{\prime} \mathrm{N}\right.$ and $\left.116^{\circ} 53^{\prime}-117^{\circ} 31^{\prime} \mathrm{E}\right)$, the southern region of Fujian province, China with an average annual rainfall of over $1600 \mathrm{~mm}$. The orchards on slopes are terraced. Most of the soils are oxisols (US Deptartment of Agriculture Soil Survey Staff) [red soil and lateritic red soil (Chinese Soil Taxonomic Classification)]. Most of the plant materials were 10 to 15 -year-old 'Guanximiyou' pummelo trees grafted on 'Sour pummelo' (Citrus grandis) rootstocks. They received similar horticultural practices, and disease and insect control.

Soil samples (one composite sample per orchard) were collected from September to October 2011. Each composite soil sample (500 - $1000 \mathrm{~g}$ per sample) was consisted of 10 sub-samples (two sub-samples per plant, five plants per orchards) taken from $0-40 \mathrm{~cm}$ depth near the canopy drip line. After air drying, the samples were gently ground, sieved ( $2 \mathrm{~mm}$ ) and properly stored for analysis (Gil et al., 2012; Lu, 1999).

Leaf samples (one composite sample per orchard) were collected from the same plants chosen for collecting soil samples from September to October 2011. Each leaf sample was consisted of 100 leaves, 20 leaves from each of five plants. The second - third leaves from the top part of the spring vegetative shoots (non-fruiting terminals) were collected from the exterior of mid canopy. Leaf samples were first washed in $0.2 \% \mathrm{HCl}$ (ca. $30 \mathrm{~s}$ ), then rinsed in tap water, finally washed in distilled water. After being wiped with towel, leaf samples were first oven-dried at $105^{\circ} \mathrm{C}$ for $30-60 \mathrm{~min}$, then at $65^{\circ} \mathrm{C}$ for $48-72 \mathrm{~h}$, ground and stored for analysis. Fruit samples (five fruits each sample, one fruit per plant) were collected from the same plants chosen for collecting soil and leaf samples during the fruit maturation (from October to November). Fruits bearing on the spring shoots were collected from the southern aspect of the exterior of mid canopy. Fruit quality parameters [i.e., fresh weight per fruit, edible rate, fruit shape index, sac granu- lation, total soluble solids (TSS), titratable acidity (TA) and $\mathrm{Vc}]$ were immediately assayed after being brought to our laboratory.

Soil analysis was performed according to $\mathrm{Lu}$ (1999) except for B. Briefly, $\mathrm{pH}$ was determined in 1: 2.5 (soil : water) soil water suspensions using $\mathrm{pH}-$ meter. $\mathrm{OM}$ was determined on the basis of oxidation with $\mathrm{K}_{2} \mathrm{Cr}_{2} \mathrm{O}_{7}$ in a heated oil bath. CEC was determined by the ammonium acetate displacement method. Hydrolysable $\mathrm{N}$ was determined with alkaline hydrolysis diffusion method. Available P was extracted from the soil with $0.5 \mathrm{M} \mathrm{NaHCO}_{3}(\mathrm{pH} 8.5)$ and determined spectrophotometrically as blue molybdate- phosphate complexes under partial reduction with ascorbic acid. Available $\mathrm{K}$, exchangeable $\mathrm{Mg}, \mathrm{Ca}$ and $\mathrm{Mn}$ were extracted from soil with $1 \mathrm{M}$ ammonium acetate (pH 7.0) and were assayed using atomic absorption spectrophotometry $(\mathrm{Ca}$, $\mathrm{Mg}$ and $\mathrm{Mn}$ ) or flame spectrophotometry (K). Available $\mathrm{S}$ was extracted with mono calcium phosphateacetic acid and determined using the simple turbidimetric method based on the formation of $\mathrm{BaSO}_{4}$ precipitate in colloid form. Available $\mathrm{Cu}$ and $\mathrm{Zn}$ were extracted by solutions of diethylene triamine pentaacetic acid (DTPA) at pH 7.3 and determined using atomic absorption spectrophotometry. Water-soluble B was extracted by hot water and measured using the curcumin method (Kowalenko and Lavkulich, 1976).

Leaf N, P and S were extracted and measured according to $\mathrm{Lu}$ (1999). Briefly, leaf $\mathrm{N}$ and $\mathrm{P}$ were assayed by semimicro distillation titration and determined colorimetrically as blue molybdate-phosphate complexes, respectively after samples being digested with $\mathrm{H}_{2} \mathrm{SO}_{4}$ and $\mathrm{H}_{2} \mathrm{O}_{2}$. Leaf $\mathrm{S}$ was determined by $\mathrm{X}$-Ray fluorescence spectrometry after samples being digested with $\mathrm{HNO}_{3}-\mathrm{HClO}_{4}$. Leaf $\mathrm{K}$, $\mathrm{Ca}, \mathrm{Mg}, \mathrm{Mn}, \mathrm{Cu}$ and $\mathrm{Zn}$ were extracted with $1 \mathrm{M} \mathrm{HCl}$ and assayed using atomic absorption spectrophotometry (Chen et al., 2011). Leaf B was measured by the curcumin method after leaf sample was ashed at $500{ }^{\circ} \mathrm{C}$ for $5 \mathrm{~h}$, and dissolved in $0.1 \mathrm{M} \mathrm{HCl}$ (Kowalenko and Lavkulich, 1976). 
TA of fruit juice was titrated with $0.1 \mathrm{~N} \mathrm{NaOH}$ to the end point $\mathrm{pH} 8.1$ using a Microprocessor-based Bench $\mathrm{pH} / \mathrm{mV} /{ }^{\circ} \mathrm{C}$ Meter ( $\mathrm{pH} 211$, Henna Instruments, Italy) and the total acidity calculated as malic acid. TSS of fruit juice was determined with WYT-4 refractometer (Quanzhou Zhongyou Optical Instrument Co., Ltd., Quanzhou, China). Vc content of fruit juice was determined according to the 2, 6-dichlorindophenol titration method (GB6195-86; a national standard determination of $\mathrm{Vc}$ in vegetables and fruits in China). Juice sac granulation degree was calculated as follow: the number of juice sac granulated segments/total number of segments $\times 100 \%$. The fruit-shape index was calculated as the ratio of fruit length to diameter. Fruit edible rate was calculated as follow: fresh weight of juice sacs/fresh weight of whole fruit $\times 100 \%$ (GB8210-87; a national standard method of inspection for export citrus fruit in China).

Range, mean, standard deviation and coefficient of variation were calculated using Microsoft Excel (Microsoft, WA, USA) spread sheet. Pearson correlation coefficient matrix was calculated using SigmaPlot 10.0 (Systat Software Inc., CA, USA).

\section{Results}

\subsection{Soil $\mathrm{pH}, \mathrm{CEC}, \mathrm{OM}$ and mineral elements}

Citrus does not like strong acid soils, because in soils of $\mathrm{pH} 5.0$ or less, serious problems may arise, such as excessive solubility of $\mathrm{Al}$ and $\mathrm{Mn}$, or low availability of $\mathrm{P}, \mathrm{Ca}, \mathrm{Mg}$ and Mo (Chapman, 1968). Soil $\mathrm{pH}$ of 319 samples ranged from 3.26 to 6.22 , with an average value of 4.34 . Out of these samples, the $\mathrm{pH}$ for $90 \%$ soils was less than 5.0 (Table 1), which is lower than the optimum range of $5.0-6.5$ for pummelo growth (Xie et al., 1997).

CEC ranged from 6.6 to $35.5 \mathrm{cmol} \mathrm{kg}^{-1}$, with an average value of $17.7 \mathrm{cmol} \mathrm{kg}^{-1}$ after (Table 1) Out of the 319 samples, the CEC value for $67.1 \%$ soils was more than $15 \mathrm{cmol} \mathrm{kg}^{-1}$, indicating that two-thirds of soils have medium or higher ability to hold positively charged ions (data not shown).

The average content of exchangeable $\mathrm{Mg}$ was $57.0 \mathrm{mg}$ $\mathrm{kg}^{-1} \mathrm{DW}$, which was less than the optimum range of 80 to $125 \mathrm{~kg}^{-1} \mathrm{DW}$ and $77.4 \%$ of soils were sub-optimum in exchangeable $\mathrm{Mg}$, followed by exchangeable $\mathrm{Ca}$ (65.8\%), hydrolysable N (48.6\%), water-soluble B (43.9\%), exchangeable $\mathrm{Mn}(29.2 \%)$ and available $\mathrm{Cu}$ (24.1\%). The average content of available $\mathrm{S}$, however, was $55.7 \mathrm{mg} \mathrm{kg}^{-1} \mathrm{DW}$, which was much more than the optimum range of 12.4 to $16.1 \mathrm{mg} \mathrm{kg}^{-1} \mathrm{DW}$ and $96.6 \%$ of soils were super-optimum in available $\mathrm{S}$, followed by available P (82.1\%), exchangeable Mn (43.3\%), available $\mathrm{Cu}(28.3 \%)$, water-soluble B (22.9\%) and OM (21.9\%) (Table 1).

Soil $\mathrm{pH}$ was negatively related with soil hydrolysable $\mathrm{N}$, available $\mathrm{P}$, exchangeable $\mathrm{Ca}$, exchangeable $\mathrm{Mg}$, available $\mathrm{S}$ or water-soluble $\mathrm{B}$, and positively related with soil $\mathrm{OM}$, available $\mathrm{Cu}$, available $\mathrm{Zn}$ or $\mathrm{CEC}$, respectively. There were positive correlations between CEC and OM as well as mineral elements except for Mn. Soil OM was positively related with soil hydrolysable $\mathrm{N}$, available $\mathrm{P}$ or exchangeable $\mathrm{Ca}$, respectively (Table 2).

\subsection{Mineral nutrient content in leaves}

Leaf $\mathrm{N}$-deficiency was the most severe among the 11 mineral elements tested and $46.8 \%$ of samples were deficient in $\mathrm{N}$, followed by $\mathrm{Mg}$ (35.6\%), P (32.3\%), $\mathrm{K}(22.3 \%)$ and $\mathrm{Cu}(14.0 \%)$. However, the average leaf content of $\mathrm{B}$ and $\mathrm{Cu}$ was 72.5 and $44.9 \mathrm{mg} \mathrm{kg}^{-1}$ $\mathrm{DW}$, which was much more than the optimum range of 15 to 50 and 8 to $17 \mathrm{mg} \mathrm{kg}^{-1} \mathrm{DW}$, respectively. Among these leaf samples tested, $74.8 \%$ and $70.3 \%$ of pummelo leaves were excess in $\mathrm{B}$ and $\mathrm{Cu}$, respec- 
tively, followed by $\mathrm{Ca}(13.4 \%), \mathrm{S}(12.1 \%)$ and $\mathrm{Mn}$ (11.4\%) (Table 3).

Leaf $\mathrm{N}$ showed a positive correlation with leaf $\mathrm{P}, \mathrm{K}$ or $\mathrm{S}$, and a negative correlation with leaf $\mathrm{Ca}$ or $\mathrm{Mg}$, respectively. Leaf $\mathrm{P}$ was positively related with leaf $\mathrm{K}, \mathrm{Mg}$ or $\mathrm{S}$, and negatively related with leaf $\mathrm{Ca}$ or
$\mathrm{K}, \mathrm{Mg}$ or $\mathrm{S}$, and negatively related with leaf $\mathrm{Ca}$ or $\mathrm{Mn}$, respectively. Leaf $\mathrm{K}$ was negatively related with leaf $\mathrm{Ca}, \mathrm{Mg}$ or $\mathrm{Mn}$, and positively related with leaf $\mathrm{S}$ or $\mathrm{Zn}$, respectively. There was no significant correlation between leaf $\mathrm{Ca}$ and $\mathrm{Mg}$ (Table 4).

Table 1. Average pH, CEC and content of OM and mineral elements in 'Guanximiyou' pummelo orchard soils, and the distribution of samples.

\begin{tabular}{|c|c|c|c|c|c|c|c|c|c|c|c|}
\hline & \multirow[t]{2}{*}{ Total samples } & \multirow[t]{2}{*}{ Range } & \multirow[t]{2}{*}{ Mean \pm SD } & \multirow{2}{*}{$\begin{array}{l}\text { Coefficient of } \\
\text { variation (\%) }\end{array}$} & \multicolumn{2}{|c|}{ Sub-optimum } & \multicolumn{2}{|l|}{ Optimum } & \multicolumn{2}{|c|}{ Super-optimum } & \multirow[t]{2}{*}{ Optimum range } \\
\hline & & & & & Samples & $\%$ & Samples & $\%$ & Samples & $\%$ & \\
\hline $\mathrm{pH}$ & 319 & $3.26-6.22$ & $4.34 \pm 0.44$ & 10.2 & 287 & 90.0 & 32 & 10.0 & 0 & 0.0 & $5.0-6.5$ \\
\hline $\mathrm{CEC}\left(\mathrm{cmol} \mathrm{kg}^{-1} \mathrm{DW}\right)$ & 319 & $6.6-35.5$ & $17.7 \pm 5.7$ & 32.0 & & & & & & & \\
\hline $\mathrm{OM}\left(\mathrm{g} \mathrm{kg}^{-1} \mathrm{DW}\right)$ & 319 & $0.65-36.79$ & $23.2 \pm 7.9$ & 34.2 & 44 & 13.8 & 205 & 64.3 & 70 & 21.9 & $15-30$ \\
\hline Hydrolysable $\mathrm{N}\left(\mathrm{mg} \mathrm{kg}^{-1} \mathrm{DW}\right)$ & 319 & $21.4-262.4$ & $104.3 \pm 35.5$ & 34.0 & 155 & 48.6 & 159 & 49.8 & 5 & 1.6 & $100-200$ \\
\hline Available P (mg kg $\left.{ }^{-1} \mathrm{DW}\right)$ & 319 & $0.5-806.2$ & $184.1 \pm 155.3$ & 84.4 & 14 & 4.4 & 43 & 13.5 & 262 & 82.1 & $10-40$ \\
\hline Available K (mg kg $\left.{ }^{-1} \mathrm{DW}\right)$ & 319 & $35.0-645.0$ & $214.2 \pm 110.6$ & 51.6 & 35 & 11.0 & 226 & 70.8 & 58 & 18.2 & $100-300$ \\
\hline Exchangeable $\mathrm{Ca}\left(\mathrm{mg} \mathrm{kg}^{-1} \mathrm{DW}\right)$ & 319 & $90.5-2705.8$ & $495.4 \pm 317.2$ & 64.0 & 210 & 65.8 & 107 & 33.5 & 2 & 0.6 & $500-2000$ \\
\hline Exchangeable $\mathrm{Mg}\left(\mathrm{mg} \mathrm{kg}^{-1} \mathrm{DW}\right)$ & 319 & $0.5-272.3$ & $57.0 \pm 48.3$ & 84.7 & 247 & 77.4 & 45 & 14.1 & 27 & 8.5 & $80-125$ \\
\hline Available S (mg kg ${ }^{-1} \mathrm{DW}$ ) & 319 & $11.0-116.7$ & $55.7 \pm 24.5$ & 43.9 & 1 & 0.3 & 10 & 3.1 & 308 & 96.6 & $12.4-16.1$ \\
\hline Water-soluble $\mathrm{B}\left(\mathrm{mg} \mathrm{kg}^{-1} \mathrm{DW}\right)$ & 319 & $0.05-4.61$ & $0.77 \pm 0.68$ & 88.9 & 139 & 43.6 & 107 & 33.5 & 73 & 22.9 & $0.5-1.0$ \\
\hline Available $\mathrm{Cu}\left(\mathrm{mg} \mathrm{kg}^{-1} \mathrm{DW}\right)$ & 315 & $0.01-29.62$ & $5.20 \pm 4.83$ & 92.9 & 76 & 24.1 & 150 & 47.6 & 89 & 28.3 & $2-6$ \\
\hline Available $\mathrm{Zn}\left(\mathrm{mg} \mathrm{kg}^{-1} \mathrm{DW}\right)$ & 319 & $1.7-32.5$ & $5.52 \pm 3.92$ & 70.9 & 8 & 2.5 & 258 & 80.9 & 53 & 16.6 & $2 \cdot 8$ \\
\hline Exchangeable $\mathrm{Mn}\left(\mathrm{mg} \mathrm{kg}^{-1} \mathrm{DW}\right)$ & 319 & $0.3-170.4$ & $11.8 \pm 17.7$ & 150.2 & 93 & 29.2 & 88 & 27.6 & 138 & 43.3 & 3-7 \\
\hline
\end{tabular}

Optimum ranges were referred to the classification standard of citrus (Xie et al., 1997; Zhuang et al., 1995).

Table 2. Pearson correlation coefficient matrix for soil $\mathrm{pH}, \mathrm{CEC}, \mathrm{OM}$ and mineral elements.

\begin{tabular}{|c|c|c|c|c|c|c|c|c|c|c|c|c|c|}
\hline & $\mathrm{pH}$ & CEC & $\mathrm{OM}$ & $\mathrm{N}$ & $\mathrm{P}$ & $\mathrm{K}$ & $\mathrm{Ca}$ & $\mathrm{Mg}$ & $\mathrm{S}$ & B & $\mathrm{Cu}$ & $\mathrm{Zn}$ & $\mathrm{Mn}$ \\
\hline $\mathrm{pH}$ & 1 & $-0.2035 * * *$ & $0.1538 * *$ & $-0.2062 * * *$ & $-0.2861 * * *$ & -0.0940 & $0.5207 * * *$ & $0.3703^{* * *}$ & $-0.3679 * * *$ & $-0.1776^{* *}$ & $0.1260^{*}$ & $0.2499 * * *$ & 0.0283 \\
\hline CEC & $-0.2035^{* * *}$ & 1 & $0.1411^{*}$ & $0.4107 * * *$ & $0.3620 * * *$ & $0.3465^{* * *}$ & $0.2512^{* * *}$ & $0.3329 * * *$ & $0.1594 * *$ & $0.2351 * * *$ & $0.1673 * *$ & $0.1323^{*}$ & 0.0306 \\
\hline OM & $0.1538 * *$ & $0.1411 *$ & 1 & $0.2915 * * *$ & $0.1691 * *$ & 0.1059 & $0.1197 *$ & 0.0932 & -0.0543 & -0.0683 & 0.0788 & 0.0071 & 0.0757 \\
\hline
\end{tabular}

*, ** and *** indicate a significant difference at $P<0.05, P<0.01$ and $P<0.001$, respectively.

Table 3. Average mineral content of 'Guanximiyou' pummelo leaves and the distribution of samples.

\begin{tabular}{|c|c|c|c|c|c|c|c|c|c|c|c|}
\hline \multirow[t]{2}{*}{ Mineral elements } & \multirow[t]{2}{*}{ Total samples } & \multirow[t]{2}{*}{ Range } & \multirow[t]{2}{*}{ Mean \pm SD } & \multirow{2}{*}{$\begin{array}{l}\text { Coefficient of } \\
\text { variation (\%) }\end{array}$} & \multicolumn{2}{|c|}{ Deficiency } & \multicolumn{2}{|c|}{ Sufficient } & \multicolumn{2}{|l|}{ Excess } & \multirow[t]{2}{*}{ Sufficient range } \\
\hline & & & & & Samples & $\%$ & Samples & $\%$ & Samples & $\%$ & \\
\hline $\mathrm{N}\left(\mathrm{g} \mathrm{kg}^{-1} \mathrm{DW}\right)$ & 316 & $14.0-31.5$ & $25.1 \pm 2.1$ & 8.5 & 148 & 46.8 & 167 & 52.8 & 1 & 0.3 & $25-31$ \\
\hline$P\left(\mathrm{~g} \mathrm{~kg}^{-1} \mathrm{DW}\right)$ & 316 & $0.87-1.93$ & $1.42 \pm 0.17$ & 11.7 & 102 & 32.3 & 211 & 66.8 & 3 & 0.9 & $1.4-1.8$ \\
\hline $\mathrm{K}\left(\mathrm{g} \mathrm{kg}^{-1} \mathrm{DW}\right)$ & 314 & $8.5-22.5$ & $15.4 \pm 2.0$ & 13.1 & 70 & 22.3 & 243 & 77.4 & 1 & 0.3 & $14-22$ \\
\hline $\mathrm{Ca}\left(\mathrm{g} \mathrm{kg}^{-1} \mathrm{DW}\right)$ & 314 & $10.3-56.3$ & $31.1 \pm 7.1$ & 22.8 & 15 & 4.8 & 257 & 81.8 & 42 & 13.4 & $20-38$ \\
\hline $\operatorname{Mg}\left(\mathrm{g} \mathrm{kg}^{-1} \mathrm{DW}\right)$ & 314 & $1.70-7.15$ & $3.51 \pm 0.88$ & 25.2 & 112 & 35.6 & 177 & 56.4 & 25 & 8.0 & $3.2-4.7$ \\
\hline $\mathrm{S}\left(\mathrm{g} \mathrm{kg}^{-1} \mathrm{DW}\right)$ & 314 & $2.41-5.33$ & $3.40 \pm 0.49$ & 14.3 & 0 & 0.0 & 276 & 87.9 & 38 & 12.1 & $2.0-3.9$ \\
\hline $\mathrm{B}\left(\mathrm{mg} \mathrm{kg}^{-1} \mathrm{DW}\right)$ & 314 & $9.6-252.0$ & $72.5 \pm 32.6$ & 44.9 & 1 & 0.3 & 78 & 24.8 & 235 & 74.8 & $15-50$ \\
\hline $\mathrm{Cu}\left(\mathrm{mg} \mathrm{kg}^{-1} \mathrm{DW}\right)$ & 313 & $2.82-180.46$ & $44.9 \pm 37.4$ & 83.3 & 40 & 14.0 & 49 & 15.7 & 220 & 70.3 & $8-17$ \\
\hline $\mathrm{Zn}\left(\mathrm{mg} \mathrm{kg}^{-1} \mathrm{DW}\right)$ & 313 & $23.1-53.8$ & $35.0 \pm 5.9$ & 16.8 & 9 & 2.9 & 281 & 89.8 & 23 & 7.3 & $24-44$ \\
\hline $\operatorname{Mn}\left(\mathrm{mg} \mathrm{kg}^{-1} \mathrm{DW}\right)$ & 316 & $5.5-365.2$ & $78.0 \pm 61.7$ & 79.1 & 6 & 1.9 & 273 & 86.7 & 37 & 11.4 & $15-140$ \\
\hline
\end{tabular}

Optimum ranges were referred to the classification standard of 'Guanximiyou' pummelo leaves (Zhuang et al., 1991) 
Table 4. Pearson correlation coefficient matrix for leaf content of mineral elements.

\begin{tabular}{|c|c|c|c|c|c|c|c|c|c|c|}
\hline & $\mathrm{N}$ & $\mathrm{P}$ & $\mathrm{K}$ & $\mathrm{Ca}$ & $\mathrm{Mg}$ & $\mathrm{S}$ & B & $\mathrm{Cu}$ & $\mathrm{Zn}$ & $\mathrm{Mn}$ \\
\hline $\mathrm{N}$ & 1 & & & & & & & & & \\
\hline $\mathrm{P}$ & $0.3241^{* * *}$ & 1 & & & & & & & & \\
\hline K & $0.1983^{* * *}$ & $0.4424 * * *$ & 1 & & & & & & & \\
\hline $\mathrm{Ca}$ & $-0.1740^{* *}$ & $-0.3583 * * *$ & $-0.4316^{* * * *}$ & 1 & & & & & & \\
\hline $\mathrm{Mg}$ & $-0.1257^{*}$ & $0.1588^{*}$ & $-0.4316^{* * * *}$ & 0.0168 & 1 & & & & & \\
\hline $\mathrm{S}$ & $0.3087 * * *$ & $0.2688^{* * *}$ & $0.2909^{* * *}$ & -0.0241 & 0.0500 & 1 & & & & \\
\hline B & -0.0534 & -0.0063 & 0.0082 & -0.0065 & $0.2014^{* * *}$ & $0.1150^{*}$ & 1 & & & \\
\hline $\mathrm{Cu}$ & 0.0139 & -0.0712 & -0.0264 & -0.0122 & $-0.1789 * *$ & -0.0579 & -0.0043 & 1 & & \\
\hline $\mathrm{Zn}$ & -0.0550 & -0.0152 & $0.1611 * *$ & -0.0969 & -0.0493 & $0.1229 *$ & 0.0498 & $-0.1343 *$ & 1 & \\
\hline $\mathrm{Mn}$ & -0.0165 & $-0.1861 * * *$ & $-0.2014 * * *$ & $0.1730^{* *}$ & 0.0740 & $-0.1664^{* *}$ & 0.0256 & 0.0121 & $0.1267^{*}$ & 1 \\
\hline
\end{tabular}

$*, * *$ and $* * *$ indicate a significant difference at $P<0.05, P<0.01$ and $P<0.001$, respectively

\subsection{Fruit quality characteristics}

We found that the coefficients of variation (CVs) for all fruit quality parameters were less than $10 \%$ except for fresh weight per fruit, fruit granulation and TSS/ TA (Table 5), meaning that fruit quality from different orchards is relatively identical, and that many of soil and leaf parameters are not related with these fruit quality parameters. There were significant relationships between most of the fruit quality parameters. For example, fruit edible rate displayed significant positive correlations with other quality parameters except for a negative correlation with TA. TSS and TA were significantly related with other parameters except for fresh weight per fruit. The best relationship existed between TSS/TA and TSS (Table 6).

Table 5. Fruit quality characteristics of 'Guanximiyou' pummelo.

\begin{tabular}{lllll}
\hline \hline & Total samples & Range & Mean \pm SD & $\begin{array}{l}\text { Coefficient of } \\
\text { variation (\%) }\end{array}$ \\
\hline Fresh weight per fruit $(\mathrm{g})$ & 304 & $467-1946$ & $1125 \pm 240$ & 21.3 \\
Edible (\%) & 304 & $40.5-76.7$ & $66.5 \pm 5.1$ & 7.6 \\
Fruit shape index & 303 & $0.77-1.19$ & $0.96 \pm 0.06$ & 6.3 \\
Fruit granulation (\%) & 304 & $4.7-78.5$ & $40.8 \pm 9.0$ & 22.1 \\
TSS (\%) & 303 & $6.7-13.4$ & $10.7 \pm 0.8$ & 8.0 \\
TA (\%) & 303 & $0.66-1.05$ & $0.83 \pm 0.06$ & 7.5 \\
TSS/TA & 302 & $6.6-19.2$ & $12.9 \pm 1.6$ & 12.4 \\
Vc (mg/100 mL) & 304 & $30.7-50.2$ & $42.4 \pm 2.4$ & 5.7 \\
\hline
\end{tabular}

Table 6. Pearson correlation coefficient matrix for parameters of fruit quality

\begin{tabular}{|c|c|c|c|c|c|c|c|c|}
\hline & $\begin{array}{l}\text { Fresh weight per } \\
\text { fruit }\end{array}$ & Edible & Fruit shape index & Fruit granulation & TSS & TA & TSS/TA & $\mathrm{Vc}$ \\
\hline $\begin{array}{l}\text { Fresh weight per } \\
\text { fruit }\end{array}$ & 1 & & & & & & & \\
\hline Edible & $0.1199^{*}$ & 1 & & & & & & \\
\hline Fruit shape index & $-0.2307 * * *$ & $0.2000^{* * *}$ & 1 & & & & & \\
\hline Fruit granulation & $0.2124^{* * *}$ & $0.3463^{* * *}$ & $0.1450^{*}$ & 1 & & & & \\
\hline TSS & -0.0397 & $0.3943^{* * *}$ & $0.2120^{* * *}$ & $0.2494 * * *$ & 1 & & & \\
\hline TA & -0.0259 & $-0.2292 * * *$ & -0.0628 & -0.0780 & $-0.3294 * * *$ & 1 & & \\
\hline $\mathrm{TSS} / \mathrm{TA}$ & -0.0068 & $0.3685^{* * *}$ & $0.1693^{* *}$ & $0.2161^{* * *}$ & $0.8306^{* * *}$ & $-0.7872^{* * *}$ & 1 & \\
\hline $\mathrm{Vc}$ & 0.0371 & $0.1408^{*}$ & 0.0469 & $0.2039^{* * *}$ & $0.2938 * * *$ & $-0.1410^{*}$ & $0.2741^{* * *}$ & 1 \\
\hline
\end{tabular}


3.4. Leaf mineral nutrients in relation to soil $\mathrm{pH}$, CEC, OM and mineral elements

Leaf $\mathrm{P}$ and $\mathrm{Ca}$ displayed positive relations with soil $\mathrm{pH}$. Leaf $\mathrm{N}$ and $\mathrm{Cu}$ were positively related with soil CEC. Leaf $\mathrm{Mg}$ had a negative relation with soil OM. Except for N, P and K, leaf Ca, Mg, S, B, Cu, Zn and Mn displayed positive relations with the corresponding soil elements, respectively (Table 7).

3.5. Fruit quality parameters in relation to soil $\mathrm{pH}$, CEC, OM and mineral nutrients

Fresh weight per fruit was negatively with soil available P. Fruit edible rate was negatively related with soil $\mathrm{OM}$ and hydrolysable $\mathrm{N}$, but positively related with soil available $\mathrm{Cu}$, respectively. Fruit shape index showed a negative relation with soil exchangeable $\mathrm{Mg}$, available $\mathrm{Zn}$ and exchangeable $\mathrm{Mn}$, respectively. Fruit TSS was negatively correlated with soil exchangeable Mg. Fruit Vc was negatively related with soil $\mathrm{pH}$ and available $\mathrm{Cu}$, respectively (Table 8).

\subsection{Fruit quality parameters in relation to leaf min- eral nutrients}

Fresh weight per fruit was positively related with leaf $\mathrm{S}$. Fruit edible rate was negatively related with leaf $\mathrm{N}$ and positively related with leaf $\mathrm{Mg}$, respectively. Juice sac granulation degree was positively related with leaf $\mathrm{Mg}, \mathrm{S}, \mathrm{Cu}$ and $\mathrm{Mn}$, respectively. TSS displayed a negative relation with leaf Ca. TA was positively related with leaf $\mathrm{S}$ and negatively related with leaf $\mathrm{Ca}$ and $\mathrm{Cu}$, respectively. TSS/TA ratio was negatively related with leaf $\mathrm{Ca}$ and positively related with leaf S, respectively (Table 9).

Table 7. Pearson correlation coefficient matrix between leaf mineral elements (first column) and soil pH, OM, CEC and mineral elements (first row)

\begin{tabular}{|c|c|c|c|c|c|c|c|c|c|c|c|c|c|}
\hline & $\mathrm{pH}$ & CEC & $\mathrm{OM}$ & $\mathrm{N}$ & $\mathrm{P}$ & $\mathrm{K}$ & $\mathrm{Ca}$ & $\mathrm{Mg}$ & $\mathrm{S}$ & B & $\mathrm{Cu}$ & $\mathrm{Zn}$ & Mn \\
\hline $\mathrm{N}$ & -0.0510 & $0.1204^{*}$ & 0.0500 & 0.0738 & -0.0248 & 0.0497 & -0.0021 & 0.0198 & $0.1162^{*}$ & -0.0586 & -0.0817 & 0.0890 & 0.0899 \\
\hline $\mathrm{P}$ & $0.1597 * *$ & -0.0174 & 0.0364 & -0.0325 & -0.0977 & -0.0538 & 0.0010 & 0.0015 & -0.0753 & -0.0905 & -0.0466 & 0.0687 & -0.0325 \\
\hline K & 0.0672 & -0.0567 & 0.0312 & 0.0135 & -0.0935 & 0.0755 & -0.0704 & -0.0534 & 0.0917 & 0.0194 & $-0.1243^{*}$ & 0.0341 & 0.0082 \\
\hline $\mathrm{Ca}$ & $0.1607^{* * *}$ & -0.0640 & 0.0109 & $-0.1314^{*}$ & 0.0285 & 0.0252 & $0.2108^{* * * *}$ & $0.1652^{* *}$ & $-0.1290^{*}$ & 0.0282 & 0.0775 & 0.0702 & 0.0783 \\
\hline $\mathrm{Mg}$ & 0.0953 & 0.0124 & $-0.1711^{* * *}$ & -0.0706 & 0.0526 & 0.0545 & 0.0273 & $0.1785^{* *}$ & -0.1094 & 0.0844 & -0.0279 & 0.0939 & -0.0571 \\
\hline S & 0.0336 & -0.0619 & 0.0032 & $-0.1334^{*}$ & $-0.1573^{* *}$ & -0.0686 & -0.0395 & -0.0009 & $0.1395^{*}$ & -0.1098 & $-0.1880^{* * *}$ & 0.0124 & -0.0650 \\
\hline B & 0.0805 & 0.0594 & -0.0972 & -0.0828 & -0.0686 & -0.0225 & 0.0145 & 0.0407 & 0.0719 & $0.2371^{* * *}$ & -0.0580 & 0.0726 & 0.0167 \\
\hline $\mathrm{Cu}$ & -0.0718 & $0.2157^{* * *}$ & -0.1092 & 0.0065 & -0.0220 & 0.0342 & -0.0284 & -0.0138 & $0.1926^{* *}$ & 0.0309 & $0.1821^{* * *}$ & -0.0643 & 0.0610 \\
\hline $\mathrm{Zn}$ & 0.0465 & -0.0819 & -0.0524 & -0.0862 & -0.0235 & -0.0287 & -0.0385 & -0.0126 & 0.0203 & $-0.1117^{*}$ & -0.0025 & $0.1180^{*}$ & 0.0418 \\
\hline $\mathrm{Mn}$ & -0.0652 & -0.0322 & -0.0657 & 0.0301 & 0.0456 & $0.1301^{*}$ & -0.0514 & 0.0840 & 0.0813 & 0.0466 & 0.0194 & 0.0657 & $0.4341 * * *$ \\
\hline
\end{tabular}

$*, * *$ and $* * *$ indicate a significant difference at $P<0.05, P<0.01$ and $P<0.001$, respectively 
Table 8. Pearson correlation coefficient matrix between soil $\mathrm{pH}, \mathrm{CEC}, \mathrm{OM}$ and mineral elements (first column) and fruit quality parameters (first row).

\begin{tabular}{|c|c|c|c|c|c|c|c|c|}
\hline & $\begin{array}{l}\text { Fresh weight per } \\
\text { fruit }\end{array}$ & Edible & Fruit shape index & Fruit granulation & TSS & TA & TSS/TA & $\mathrm{V}_{\mathrm{c}}$ \\
\hline $\mathrm{pH}$ & 0.0583 & -0.0430 & -0.0545 & 0.0035 & -0.0661 & 0.0196 & -0.0529 & $-0.1328 *$ \\
\hline CEC & -0.0327 & -0.1099 & -0.0160 & -0.0376 & -0.0251 & -0.0401 & 0.0030 & -0.0213 \\
\hline OM & -0.0382 & $-0.1180^{*}$ & -0.0356 & -0.0973 & -0.1003 & -0.0206 & -0.0549 & 0.0116 \\
\hline $\mathrm{N}$ & -0.0574 & $-0.1189 *$ & -0.0545 & -0.0483 & -0.0387 & 0.0068 & -0.0246 & 0.0276 \\
\hline $\mathrm{P}$ & $-0.1201^{*}$ & 0.0938 & -0.1099 & 0.0093 & -0.0497 & -0.0265 & -0.0196 & 0.0061 \\
\hline K & -0.0635 & -0.0320 & -0.0953 & 0.0018 & -0.1096 & 0.0520 & -0.1102 & 0.0316 \\
\hline $\mathrm{Ca}$ & -0.0058 & -0.0022 & -0.1016 & -0.0307 & -0.1003 & -0.0490 & -0.0406 & -0.0332 \\
\hline $\mathrm{Mg}$ & 0.0422 & -0.0177 & $-0.1552^{* *}$ & -0.0091 & $-0.1152 *$ & -0.0167 & -0.771 & 0.0108 \\
\hline S & 0.0533 & -0.0302 & 0.0218 & 0.0206 & 0.0867 & 0.0179 & 0.0383 & 0.1114 \\
\hline B & -0.0567 & 0.0357 & 0.0103 & -0.0144 & -0.0012 & 0.0770 & -0.0487 & 0.0102 \\
\hline $\mathrm{Cu}$ & -0.0912 & $0.1181^{*}$ & 0.0283 & 0.0729 & -0.1023 & 0.0519 & -0.1024 & $-0.1303 *$ \\
\hline $\mathrm{Zn}$ & -0.0112 & 0.0506 & $-0.1375^{*}$ & 0.0271 & -0.0922 & 0.0123 & -0.0756 & -0.0683 \\
\hline $\mathrm{Mn}$ & 0.0400 & -0.1113 & $-0.1176^{*}$ & 0.0359 & -0.0940 & 0.0441 & -0.0876 & 0.0647 \\
\hline
\end{tabular}

*,** and *** indicate a significant difference at $P<0.05, P<0.01$ and $P<0.001$, respectively

Table 9. Correlation coefficient matrix between leaf mineral elements (first column) and fruit quality parameters (first row)

\begin{tabular}{|c|c|c|c|c|c|c|c|c|}
\hline & $\begin{array}{l}\text { Fresh weight per } \\
\text { fruit }\end{array}$ & Edible & Fruit shape index & Fruit granulation & TSS & TA & TSS/TA & $\mathrm{Vc}_{\mathrm{c}}$ \\
\hline $\mathrm{N}$ & 0.0780 & $-0.1447 *$ & -0.1039 & -0.0783 & -0.0548 & 0.0385 & -0.0535 & -0.0372 \\
\hline$P$ & 0.1083 & 0.0260 & -0.0829 & -0.0301 & -0.0156 & -0.1098 & 0.0547 & -0.0773 \\
\hline K & 0.0034 & -0.0771 & 0.0122 & 0.0388 & 0.0102 & -0.0816 & 0.0607 & 0.0679 \\
\hline $\mathrm{Ca}$ & 0.0153 & 0.0328 & -0.0178 & 0.0377 & $-0.1588 * *$ & $0.1217^{*}$ & $-0.1852 * *$ & 0.0497 \\
\hline $\mathrm{Mg}$ & 0.0991 & $0.1525^{* *}$ & -0.0137 & $0.1199 *$ & 0.0440 & -0.0337 & 0.0465 & 0.0028 \\
\hline $\mathrm{S}$ & $0.1749 * *$ & 0.0741 & 0.0136 & $0.1348^{*}$ & 0.0923 & $-0.1843^{* *}$ & $0.1570^{* *}$ & 0.1109 \\
\hline B & 0.0291 & 0.0234 & -0.0187 & -0.0091 & 0.0042 & -0.0269 & 0.0184 & 0.0728 \\
\hline $\mathrm{Cu}$ & -0.0984 & -0.0798 & 0.0768 & $0.1711^{* *}$ & -0.0107 & $0.1796^{* *}$ & -0.1040 & 0.0010 \\
\hline $\mathrm{Zn}$ & -0.0234 & 0.1104 & -0.0268 & 0.0124 & 0.0721 & -0.0643 & 0.0774 & -0.0058 \\
\hline $\mathrm{Mn}$ & 0.0485 & -0.0283 & -0.0879 & $0.1266^{*}$ & 0.0176 & 0.1104 & -0.0596 & 0.0472 \\
\hline
\end{tabular}

*,** and *** indicate a significant difference at $P<0.05, P<0.01$ and $P<0.001$, respectively

\section{Discussion}

\subsection{Soil acidification is a major problem in 'Guanxi- miyou' pummelo orchard soils}

Although citrus (pummelo) can be planted in soils with either a high or low $\mathrm{pH}$, the optimum $\mathrm{pH}$ ranges from 5.0 to 6.5 (Xie et al., 1997). Rasmussen and Smith (1957) grew Pineapple orange seedlings in combined soils with different $\mathrm{pH}$ levels (i.e., 4.0, 4.3, 4.6, 5.0, and 6.0). Five months after growing, growth was the best under $\mathrm{pH} 6.0$ and was considerably poorer at $\mathrm{pH}$ 4.0. In this study, the lowest, average and highest $\mathrm{pH}$ values of the 319 soils were $3.26,4.34$ and 6.22 , respectively, which were lower than the previous values (i.e., 3.57, 4.63 and 7.25; Huang et al., 2001). In addition, up to $21.0 \%$ soils had a $\mathrm{pH}$ of less than 4.0 (data not shown). Obviously, soil $\mathrm{pH}$ is rapidly de- 
creasing in the last decade. Under natural conditions, soils acidify very slowly over hundreds to millions of years (Guo et al., 2010). Rapid acidification of Pinghe pummelo orchard soils might be mainly caused by improper fertilization. In Pinghe, orchard farmers usually overuse compound fertilizer containing $15 \%$ $\mathrm{N}, 15 \% \mathrm{P}_{2} \mathrm{O}_{5}$ and $15 \% \mathrm{~K}_{2} \mathrm{O}$ and physiological acidic fertilizers such as $\mathrm{Mg}_{2} \mathrm{SO}_{4}$, and seldom apply basic fertilizers such as lime and $\mathrm{Mg}(\mathrm{OH})_{2}$. In addition, the environmental problems such as acid rain and some agriculture practices also contribute to the acidification of these soils (Guo et al., 2010).

\subsection{Severe soil and leaf nutrient imbalance}

Organic manures were heavily applied in recent years. As expected, the average content of soil OM (23.2 g $\left.\mathrm{kg}^{-1} \mathrm{DW}\right)$ was higher than the previous value $(17.7 \mathrm{~g}$ $\mathrm{kg}^{-1} \mathrm{DW}$ ), and the percentage of sub-optimum soils (13.8\%) was lower than the previous report (29.5\%) (Table 1; Huang et al., 2001). Soil OM increases soil fertility by providing cation exchange sites and acting as reserve of essential nutrients, especially $\mathrm{N}$ and $\mathrm{P}$, which are slowly released upon soil OM mineralization (Tarrasón et al., 2007). This agrees with our data that soil CEC, hydrolysable N, available P and exchangeable $\mathrm{Ca}$ were positively related with soil $\mathrm{OM}$ (Table 2). It has been known that strong acidification can be buffered by OM (Aitken et al., 1990). The negative relationship between soil OM and $\mathrm{pH}$ (Table 2) means that soil acidification caused by improper fertilization and environmental problems can not be completely buffered by increased OM.

Our finding that soil CEC was negatively related with $\mathrm{pH}$ (Table 2) agrees with the view that as soil $\mathrm{pH}$ decreases, more $\mathrm{H}^{+}$ions are attached to the colloids and push other cations from the colloids and into the soil solution (CEC decreases). The optimum soil CEC for citrus ranges from 12 to $16 \mathrm{cmol} \mathrm{kg}^{-1} \mathrm{DW}$ (Li et al.,
2001). Based on the criterion, only $11.9 \%$ soil samples had sub-optimum CEC. This disagrees with our data that $77.4 \%$ and $65.8 \%$ of soils had sum-optimum $\mathrm{Ca}$ and $\mathrm{Mg}$, respectively (Table 1) and soil CEC was positively related with exchangeable $\mathrm{Ca}$ and $\mathrm{Mg}$ (Table 2). Soil OM has a particularly high CEC ranging from 250 to $400 \mathrm{cmol} \mathrm{kg}^{-1} \mathrm{DW}$, and can account for 30 - $60 \%$ or more of CEC (Loveland and Webb, 2003). Our results showed that soil CEC was positively correlated with soil OM (Table 2), and that only $13 \%$ of soils had sub-optimum OM (Table 1). Thus, it is reasonable to assume that the differences between soil $\mathrm{CEC}$ and exchangeable $\mathrm{Ca}$ and $\mathrm{Mg}$ are caused mainly by the relatively higher soil OM (Table 1).

Like that of the previous workers (Huang et al., 2001), our results showed that soil sub-optimum $\mathrm{Mg}$ and $\mathrm{Ca}$ were the two most widespread nutrient constraints in pummelo orchard soils (Table 1). Soil exchangeable $\mathrm{Ca}$ and $\mathrm{Mg}$ were positively related with soil $\mathrm{pH}$ (Table 2). Thus, it is reasonable to assume that soil acidification might be the major factor contributing to low levels of soil exchangeable $\mathrm{Mg}$ and $\mathrm{Ca}$. It is worth noting that sub-optimum $\mathrm{Mg}$ soils (77.4\%) was higher than sub-optimum $\mathrm{Ca}$ ones $(65.8 \%$; Table 1). Poss and Saragoni (1992) showed that exchangeable $\mathrm{Mg}$ was less adsorbed by the solid phase of the soil (xoisol) than exchangeable $\mathrm{Ca}$, and that exchangeable $\mathrm{Mg}$ was preferentially leached from the upper layers of the soil than the exchangeable $\mathrm{Ca}$. This can, at least in part, explain the higher percentage of sub-optimum $\mathrm{Mg}$ in 'Guanximiyou' pummelo orchards with heavy application of $\mathrm{Mg}$ fertilizers.

The average content of soil hydrolysable $\mathrm{N}$ was 104.3 $\mathrm{mg} \mathrm{kg}{ }^{-1} \mathrm{DW}$, which was lower than the previous report (121.0 $\mathrm{mg} \mathrm{kg}^{-1} \mathrm{DW}$ ), and $48.6 \%$ of soils was suboptimum in hydrolysable $\mathrm{N}$, which was higher than the previous report (44.0\%; Huang et al., 2001). This means that soil hydrolysable $\mathrm{N}$ sub-optimum status was not improved over the last decade. According to 
our investigation, most of orchards were applied with sufficient $\mathrm{N}$ fertilizers (600 $\mathrm{kg} \mathrm{ha}^{-1}$ or more per year). The observed increase in $\mathrm{N}$ sub-optimum soils might be caused by low $\mathrm{N}$ use efficiency due to improper application such as broadcasting. Leaching of $\mathrm{N}$ in the nitrate form is a very important factor leading to soil acidity (Huang et al., 2014). Soil acidification in recent years implies that $\mathrm{N}$ leaching may be increased, which may contribute to the low availability of soil $\mathrm{N}$. Available $\mathrm{S}$ content in $99.6 \%$ of soils was higher than the optimum range (Table 1). This might be mainly caused by the long-term and heavy application of $\mathrm{MgSO}_{4}$. Application of $2 \mathrm{~kg} \mathrm{MgSO}_{4} \cdot 7 \mathrm{H}_{2} \mathrm{O}$ tree ${ }^{-1}$ year ${ }^{-1}$ is very common for most of orchards. In addition, the application of fungicides containing $\mathrm{S}$ (i.e., lime sulphur) may contribute to the accumulation of soil available $\mathrm{S}$.

Available P content in $82.1 \%$ of soils was higher than the optimum range (Table 1), which was higher than the previous result (70.5\%). Also, the average content of soil available $\mathrm{P}\left(184.1 \mathrm{mg} \mathrm{kg}^{-1} \mathrm{DW}\right)$ was much more than the previous report $\left(112.3 \mathrm{mg} \mathrm{kg}^{-1}\right.$ DW; Huang et al., 2001). The observed increase in soil available $\mathrm{P}$ might be caused by the heavy application of compound fertilizer containing $15 \% \mathrm{~N}$, $15 \% \mathrm{P}_{2} \mathrm{O}_{5}$ and $15 \% \mathrm{~K}_{2} \mathrm{O}$.

We found that $46.8 \%$ of leaf samples were deficient in $\mathrm{N}$, which were much higher than the previous result $(7.0 \%)$, and that the average content of leaf $\mathrm{N}$ (25.1 $\left.\mathrm{g} \mathrm{kg}^{-1} \mathrm{DW}\right)$ was lower than the previous result (28.6 $\left.\mathrm{g} \mathrm{kg}^{-1} \mathrm{DW}\right)$ (Table 3; Huang et al., 2001). Obviously, N-deficiency was becoming more and more widespread. Although the degrees of soil $\mathrm{N}$ sub-optimum (48.6\%) and leaf $\mathrm{N}$-deficiency were basically similar (Tables 1 and 3), the relationship between leaf $\mathrm{N}$ and soil available $\mathrm{N}$ was poor (Table 7). However, Yu et al. (2007) reported that Ponkan (Citrus reticulata) leaf $\mathrm{N}$ was positively related with soil hydrolysable N.
Although only $4.4 \%$ of and up to $82.1 \%$ of soils were sub-optimum and super-optimum in available $\mathrm{P}$, respectively (Table 1), up to $32.3 \%$ of and only $0.9 \%$ of leaf samples were deficient and excess in $\mathrm{P}$, respectively (Table 2 ). This agrees with our data that leaf $\mathrm{P}$ was not related with soil available P (Table 7). Thus, leaf P-deficiency was not mainly caused by lower soil available $\mathrm{P}$ and other factors might contribute to the exacerbation of leaf P-deficiency. Also, no significant relation was observed between leaf $\mathrm{P}$ and soil available P on 'Guanximiyou' pummelo (Huang et al., 2001), C. sinensis (Tang et al., 2013) and Ponkan (Yu et al., 2007). This demonstrates the complexes of citrus $\mathrm{P}$ uptake.

We found that leaf $\mathrm{K}$ was not related with soil available K (Table 7), as previously reported on Ponkan (Yu et al., 2007), 'Guanximiyou' pummelo (Huang et al., 2001) and C. sinensis (Tang et al., 2013). In our study, $11.0 \%$ and $18.2 \%$ of orchard soils were sub-optimum and super-optimum in soil available $\mathrm{K}$, respectively (Table 1), while up to $22.3 \%$ and only $0.3 \%$ of leaf samples were deficient and excess in leaf $\mathrm{K}$, respectively (Table 3 ). Therefore, soil available $\mathrm{K}$ level was not the major factor affecting leaf $\mathrm{K}$ content.

In China, leaf Mg-deficiency is frequently observed in citrus plantations, including 'Guanximiyou' pummelo orchards (Huang et al., 2001; Zhuang, 1994). As expected, $35.6 \%$ of leaves were deficient in $\mathrm{Mg}$ (Table 3). Regressive analysis showed that soil exchangeable $\mathrm{Mg}$ was positively related with soil $\mathrm{pH}$ (Table 2), and that leaf $\mathrm{Mg}$ was positively related with soil exchangeable $\mathrm{Mg}$ (Table 7). This drove us to conclude that soil acidification lowered soil exchangeable $\mathrm{Mg}$ level, thus reducing $\mathrm{Mg}^{2+}$ uptake, eventually resulting in leaf Mg-deficiency. However, only $4.8 \%$ of leaf samples were deficient in Ca (Table 3 ), although $65.8 \%$ of soils were sub-optimum in soil exchangeable $\mathrm{Ca}$ (Table 1). Furthermore, 13.4\% 
of leaf samples were excess in Ca (Table 3), although only $0.6 \%$ of soil samples were super-optimum in exchangeable $\mathrm{Ca}$ (Table 1). The antagonism between $\mathrm{Ca}$ and $\mathrm{Mg}$ for uptake has been known in citrus (Moss and Higgins, 1974). This is also supported by our data that leaf $\mathrm{Ca}$ and $\mathrm{Mg}$ were negatively related with leaf $\mathrm{Mg} / \mathrm{Ca}$ and $\mathrm{Ca} / \mathrm{Mg}$ ratio, respectively (data not shown). Thus, the difference between leaf $\mathrm{Ca}$ and soil exchangeable $\mathrm{Ca}$ can be explained in this way. Although $43.6 \%$ of soils were sub-optimum in soil water-soluble B (Table 1), up to $74.8 \%$ of leaf samples were excess in B (Table 3). This agrees with the previous result obtained on 'Guanximiyou' pummelo (Huang et al., 2001). Our results showed that there was a positive between leaf B and soil water-soluble B (Table 7). However, Huang et al. (2001) showed that leaf B did not correlate with soil water-soluble B. Based on these results, we concluded that the observed higher leaf B excess might be mainly caused by overuse of B fertilizer through frequent foliar application rather than by high soil water-soluble B. Similarly, leaf $\mathrm{Cu}$ excess was more pronounced than soil super-optimum in available $\mathrm{Cu}$ (Tables 1 and 3). The observed higher leaf $\mathrm{Cu}$ excess might be mainly due to application of fungicides containing $\mathrm{Cu}$ (i.e., Bordeaux mixture).

To conclude, severe nutrient imbalance existed in 'Guanximiyou' pummelo orchard soils and leaves. Leaf content of mineral elements did not highly correlate with the available content of the corresponding soil elements, respectively.

\subsection{Effects of mineral nutrients on fruit quality}

Previous studies showed that the nutritional status of plants played a role in citrus granulation (Wang et al., 2014). Our result showed that the degree of juice sac granulation was positively related with leaf S (Table 9). This agrees with our previous report that $\mathrm{S}$ content was higher in granulated juice sacs of 'Guanximiyou' pummelo fruits than in normal ones (Wang et al., 2014). This suggests that $\mathrm{S}$ might play a role in the juice sac granulation of pummelo fruits. However, no relationship was found between leaf $\mathrm{S}$ and the incidence of granulation in sweet orange (Awasthi and Nauriyal, 1972).

We observed a positive relationship between leaf $\mathrm{Cu}$ and juice sac granulation of 'Guanximiyou' pummelo fruits (Table 9), which agrees with the reports that leaf $\mathrm{Cu}$ increased as the fruit granulation developed in two sweet orange cultivars (Munshi et al., 1978), and that granulated juice sacs of 'Guanximiyou' pummelo fruits had higher $\mathrm{Cu}$ content compared with normal ones (Wang et al., 2014). Thus, $\mathrm{Cu}$ might be involved in the juice sac granulation of 'Guanximiyou' pummelo fruits. However, juice sac granulation was not significantly related with leaf $\mathrm{Cu}$ in 'Guanximiyou' pummelo (Xie et al., 1998) and sweet orange (Awasthi and Nauriyal, 1972). In addition, no significant difference was found in $\mathrm{Cu}$ content between normal and granulated juice sacs of 'Guanximiyou' pummelo fruits (Xie et al., 1998).

Juice sac granulation was positively related with leaf $\mathrm{Mg}$. This agrees with the previous reports that $\mathrm{Mg}$ content was higher in granulated juice sacs than in normal ones of 'Guanximiyou' pummelo (Wang et al., 2014; Xie et al, 1998) and 'Valencia' orange (Sinclair and Jolliffe, 1961) fruits. However, no significant relationship was found between leaf $\mathrm{Mg}$ content and juice sac granulation in sweet orange (Awasthi and Nauriyal, 1972).

Our finding that juice sac granulation was positively related with leaf Mn (Table 9) disagrees with the previous result obtained on sweet orange (Awasthi and Nauriyal, 1972; Munshi et al., 1978). In addition, Mn content did not significantly differ between normal and granulated juice sacs of 'Guanximiyou' pummelo fruits (Wang et al., 2014). 
Fruit edible rate was negatively related with leaf $\mathrm{N}$, but positively related with leaf $\mathrm{Mg}$ (Table 9). This agrees with the previous reports that $\mathrm{N}$ increased citrus peel thickness (Chapman, 1968; Zhuang, 1994), and that $\mathrm{Mg}$ decreased citrus rind thickness (Zekri et al., 2009).

We found that TSS and TA were negatively and positivily related with leaf Ca respectively (Table 9), which disagrees with the results that Ca-deficiency increased TA and decreased TSS of citrus fruits (Zhuang, 1994). In addition, fruit TA was positively related with leaf $\mathrm{Cu}$ (Table 9), which agrees with the result that $\mathrm{Cu}$-deficiency decreased the TA of orange juice (Chapman, 1968). TA displayed a negative relation with leaf $\mathrm{S}$ (Table 9). Our previous results showed that TA was lower in granulated juice sacs of 'Guanximiyou' pummelo fruits than in normal ones, while $\mathrm{S}$ content was higher in the former (Wang et al., 2014), and that juice sac granulation was positively related with leaf $\mathrm{S}$ (Table 9). Thus, it is reasonable to assume that S-induced decrease in TA may be caused by increasing juice sac granulation.

\section{Conclusion}

Soil acidification is a major problem in 'Guanximiyou' pummelo orchards. Soil acidification affected the availability and uptake of mineral nutrient elements, thus inducing severe soil and leaf nutrient imbalance, eventually impairing fruit quality parameters such sac granulation.

\section{Acknowledgement}

This work was financially supported by the earmarked fund for China Agriculture Research System.

\section{References}

Aitken, R.L., Moody, P.W., McKinley, P.G. 1990. Lime requirement of acidic Queensland soils. I. relationships between soil properties and $\mathrm{pH}$ buffer capacity. Aust. J. Soil Res. 28, 695-701.

Awasthi, R., Nauriyal, J. 1972. Studies on nutritional status of trees and carbohydrate metabolism in relation to granulation in sweet orange. Indian J. Hort. 29, 252-257.

Chapman, H.D. 1968. The mineral nutrition of citrus. In: W. Reuther, H.J. Webber, L.D. Batchelor (eds). The Citrus Industry, Vol. 2. Division of Agricultural Sciences, University of California, Berkeley, CA, USA, pp. 127-89.

Chen, A.H., Jiao, B.N., Wang, C.Q. 2011. Extraction of mineral elements from citrus leaves with boiling hydrochloric acid. J. Fruit Sci. 28, 1107-1110.

Du Plessis, S.F. 1977. Soil analysis as a necessary complement to leaf analysis for fertilizer advisory purposes. Proc. Int. Soc. Citriculture 1, 15-19.

Gill, P.M., Bonomelli, C., Schaffer, B., Ferreyra, R., Gentina, C. 2012. Effect of soil water-to-air ratio on biomass and mineral nutrition of avocado trees. J. Soil Sci. Plant Nutr. 12, 609-630.

Guo, J.H., Liu, X.J., Zhang, Y., Shen, J.L., Han, W.X., Zhang, W.F., Christie, P., Goulding, K.W., Vitousek, P.M., \& Zhang, F.S. 2010. Significant acidification in major Chinese croplands. Science 327, 1008-1010.

Huang, Y.Z., Li, J., Wu, S.H., Pang, D.M. 2001. Nutrition condition of the orchards in the main production areas of Guanxihoney pomelo trees (Pinhe county). J. Fujian Agri. Univ. 30, 40- 43.

Huang, J., Mo, J.M., Zhang, W., Lu, X.K. 2014. Research on acidification in forest soil driven by atmospheric nitrogen deposition. Acta Ecol. Sin. 34, 302-310. 
Kowalenko, C.G., Lavkulich, L.M. 1976. A modified curcumin method for boron analysis of soil extracts. Can. J. Soil Sci. 56, 537-539.

Li, Z.L., Wang, C.Q., Wang, S.L., Xie, D.T., Wei, C.F. 2001. Citrus growth and soil environment. South China Fruits 30(3), 17-21.

Loveland, P., Webb, J. 2003. There a critical level of organic matter in the agricultural soils of temperate regions: a review. Soil Till. Res. 70, 1-18.

Lu, R.K. 1999. Methods of Soil and Agrochemistry Analysis. China Agriculture Science \& Technology Press, Beijing, China.

Lu, Y.M. 2013. Occurrence and control of 'Guanximiyou' foot rot disease in Pinhe county. Southeast Hort. (4), 27-28

Ministry of Agriculture P.R.C. 2012. China Agriculture Statistical Report 2011. China Agriculture Press, Beijing, China.

Moss, G.I., Higgins, M.L. 1974. Magnesium influences on the fruit quality of sweet orange (Citrus sinensis L. Osbeck). Plant Soil 41, 103-112.

Munshi, S.K., Singh, R., Vij, V.K., Jawanda, J.S. 1978. Mineral composition of leaves in relation to degree of granulation in sweet orange. Sci. Hort. 9, 357-367.

Poss, R., Saragoni, H. 1992. Leaching of nitrate, calcium and magnesium under maize cultivation on an oxisol in Togo. Fert. Res. 33, 123-133.

Rasmussen, G. K., Smith, P. F. 1957. Effect of subsoil type and $\mathrm{pH}$ on growth and macronutrient level of leaf tissue of Pineapple orange seedlings. Soil Crop Sci. Soc. Fla. Proc. 17, 243-248.

Sinclair, W.B., Jolliffe, V.A. 1961. Chemical changes in the juice vesicles of granulated valencia oranges. J. Food Sci. 26, 276-282.

Tang, Y.Q., Peng, L.Z., Chun, C.P., Ling, L.L., Fang, Y.W., Yang, X. 2013. Correlation analysis on nutrient element contents in orchard soils and sweet orange leaves in southern Jiangxi province of China. Acta Hort. Sin. 40, 623-632.

Tarrasón, D., Ortiz, O., Alcañiz, J.M. 2007. A multicriteria evaluation of organic amendments used to transform an unproductive shrubland into a Mediterranean dehesa. J. Environ. Manage. 82, 446-456.

Wang, X.Y., Wang, P., Qi, Y.P., Zhou, C.P., Yang, L.T., Liao, X.Y., Wang, L.Q., Zhu, D.H., Chen, L.S. 2014. Effects of granulation on organic acid metabolism and its relation to mineral elements in Citrus grandis juice sacs. Food Chem. 145, 894990.

Xie, Z.N., Zhuang, Y.M., Wang, R.J., Xu, W.B. 1997. Correlation between soil $\mathrm{pH}$ and the contents of available nutrients in selected soils from three kinds of orchards at subtropical zone in Fujian. Acta Hort. Sin. 24, 209-214.

Xie, Z.N., Zhuang, Y.M., Wang, R.J., Xu, W.B., Huang Y.Z. 1998. Granulation and dehiscent segments of Guanxi honey pomelo fruits and their correlation to mineral nutrients. J. Fujian Agri. Univ. 27, 42-46.

Yu, H.B., Wang, R.C., Xiao, R.L., Yang, Z. 2007. Soil and leaf nutrient condition of plants from citrus orchards in demonstration area of environmental immigrants, northwest Guangxi province. J. Hunan Agri. Univ. (Nat. Sci.) 33, 341-344,357.

Zekri, M., Obreza, T.A., Koo, R. 2009. Irrigation, nutrition, and citrus fruit quality. SL 207, Florida Cooperative Extension Service, Institute of Food and Agricultural Sciences, University of Florida.

Zhuang, Y.M., Wang, R.J., Chen, L.X., Xu, W.B., Huang, Y.Z., Zhou, Z.L. 1991. Optimum range of mineral element content in the leaves of Guanxi honey pumelo (Citrus grandis). J. Fujian Acad. Agri. Sci. 6(2), 52-58.

Zhuang, Y.M. 1994. Citrus Nutrition and Fertilizeration. China Agriculture Press, Beijing, China. 
Zhuang, Y.M., Wang, R.J., Xie, Z.N., Xu, W.B. 1995.

Study on nutrition diagnosis standard of citrus, longan and litchi. Fujian Fruits (1), 6-9. 